\title{
Audit of acute Charcot's disease in the UK: the CDUK study
}

\author{
F. L. Game • R. Catlow • G. R. Jones • M. E. Edmonds • \\ E. B. Jude • G. Rayman • W. J. Jeffcoate
}

Received: 13 June 2011 / Accepted: 3 October 2011 / Published online: 8 November 2011

(C) Springer-Verlag 2011

\begin{abstract}
Aims/hypothesis We studied factors associated with the development and resolution of acute Charcot foot using a web-based observational study.

Methods Clinicians managing cases of acute Charcot foot in the UK and Ireland between June 2005 and February 2007 were invited to register anonymised details on a secure website.

Results A total of 288 cases (age $57.0 \pm 11.3$ years $[$ mean \pm SD]; $71.2 \%$ male) were registered from 76
\end{abstract}

Electronic supplementary material The online version of this article (doi:10.1007/s00125-011-2354-7) contains a peer-reviewed but unedited list of participating centres as supplementary material, which is available to authorised users.

\section{F. L. Game $(\bowtie) \cdot$ W. J. Jeffcoate}

Foot Ulcer Trials Unit,

Department of Diabetes and Endocrinology,

Nottingham University Hospitals Trust,

City Hospital Campus, Hucknall Road,

Nottingham NG5 1PB, UK

e-mail: fgame@futu.co.uk

R. Catlow $\cdot$ G. R. Jones

East Lancashire Healthcare Trust,

Blackburn, UK

M. E. Edmonds

Kings College Hospital,

London, UK

E. B. Jude

Tameside General Hospital,

Tameside, UK

G. Rayman

Ipswich Hospital,

Ipswich, UK centres. Of these, $36 \%$ of patients recalled an episode of relevant trauma in the preceding 6 months, while $12 \%$ had had surgery to the affected foot. In $101(35 \%)$ cases, ulceration was present at registration and $20 \%$ of these had osteomyelitis. Non-removable off-loading devices were used at presentation in $35.4 \%$ of cases, with removable off-loading used in $50 \%$. Data on resolution were available for 219 patients. The median time to resolution was 9 months in patients whose initial management included the use of non-removable off-loading, compared with 12 months in the remainder $(p=0.001)$. Bisphosphonates were administered intravenously in $25.4 \%$ and orally in $19.4 \%$ of cases. The median time to resolution in patients who received bisphosphonates was 12 months and was longer than in those who did not (10 months, $p=0.005$ ).

Conclusions/interpretation The median time to resolution was longer than in earlier series. Although limited by being observational and non-randomised, these data suggest that the use of non-removable off-loading at presentation may shorten the time to resolution. They provide no evidence to indicate that the use of bisphosphonates is beneficial.

Keywords Bisphosphonates - Charcot foot - Diabetic foot Neuro-osteoarthropathy

\section{Abbreviation \\ RCT Randomised controlled trial}

\section{Introduction}

Acute Charcot's disease (Charcot foot; neuropathic osteoarthropathy) is an uncommon complication of distal symmetrical neuropathy, and is characterised by fracture 
and dislocation of the bones and joints of the foot [1-3]. In some cases the structural damage is limited, but in others it is progressive and extensive, leading to deformity, secondary ulceration and osteomyelitis, which can be difficult or impossible to eradicate. Although uncommon, acute Charcot's disease is a source of major suffering and cost.

The pathogenesis of Charcot's disease is not known, but it is thought that a subgroup of patients with distal symmetrical neuropathy is at risk and that the condition is triggered in this group by local inflammation [4-6]. This inflammation may be caused by minor trauma, surgery, infection or earlier ulceration, and the possibility of a genetic predisposition to abnormal signalling through inflammatory pathways has been suggested [7].

Diagnosis is largely dependent on pattern recognition by an experienced clinician and rests on the presence of unexplained inflammation in a foot affected by distal symmetrical neuropathy, with or without fracture and/or dislocation of the bones and joints. If necessary, diagnosis is confirmed by the demonstration of oedema of the bone marrow on MRI.

It is generally accepted that off-loading in a non-removable below-knee cast or cast-walker is an essential part of management [4]. In addition, the use of pamidronate was shown in an open-label study to reduce skin temperature and bone turnover markers [8], the latter effect being subsequently confirmed in a randomised controlled trial (RCT) [9]. Another small RCT demonstrated that weekly treatment with alendronate also led to a significant reduction in bone turnover markers, associated with reduced discomfort [10]. Despite these observations, the long-term clinical benefit of non-removable off-loading or bisphosphonate therapy has not yet been clearly established.

To address these uncertainties, we undertook a webbased survey of new cases of acute Charcot foot at centres in the UK and Ireland over a period of 20 months. This survey was designed to confirm the demographics, explore possible causative factors, record the time taken to apparent resolution and determine whether time to resolution was associated with any particular intervention.

\section{Methods}

Clinicians caring for patients with acute Charcot's disease of the foot were alerted to the study by fliers at specialist meetings, advertisement in specialist journals and through clinical networks, and asked to register anonymised details of all newly presenting or currently active cases of acute Charcot's disease on a secure website. No specific diagnostic criteria were applied and the clinician's decision or diagnosis was accepted. Details of demographics, approximate date of onset of the acute episode and preceding events (such as foot surgery, ulceration, infection or trauma) were requested, as well as details of treatments given, including types of off-loading and the use of bisphosphonates. Computerised prompts were used to request follow-up information at intervals of 3 months up to 18 months after registration. The date of resolution was predefined as the time when the patient was ambulant in normal or orthotic footwear. Data were analysed using SPSS version 17 (SPSS, Chicago, IL, USA). As this was an observational study of anonymised data collected as part of routine management, formal ethical approval was not required.

\section{Results}

We registered 288 patients from 76 different centres in the UK and Ireland in the 20 months between June 2005 and February 2007. The numbers of patients registered per centre ranged from 1 to 22 , with a median of 3.0. Median time from onset of the disease to registration was 4 months (range 0-28 months). Of patients registered, 70\% had type 2 diabetes and $71.2 \%$ were male. Mean age $( \pm \mathrm{SD})$ was $57.0 \pm 11.3$ years (men $57.9 \pm 10.8$, range 25 85 years; women $54.5 \pm 12.2$, range $21-90$ years). The majority of lesions were in the mid and hind foot (digits $1.8 \%$, digits and metatarsals $2.9 \%$, metatarsals $10.2 \%$, metatarsals and tarsals $30.7 \%$, tarsals $45.3 \%$, tarsals and ankle $8.7 \%$ ). Previous episodes of acute Charcot foot were reported to have occurred in $15 \%$ of patients, and two patients had previous bilateral disease. An episode of trauma was recalled by $104(36 \%)$ patients, while 35 (12\%) patients had had some surgical intervention to the index limb within the 6 months prior to registration. A total of $101(35 \%)$ patients had an ulcer on the affected limb, complicated by osteomyelitis in 20 of these $(7 \%$ of 288). Follow-up data were available on 219 patients $(76.0 \%)$ in whom the acute phase of the disease resolved during the study, with a median time to resolution (ambulant in orthotic or normal shoes) of 10.0 months (range 2-40 months). Ulceration was present on the acute Charcot foot in $35 \%$ of patients at registration, and in $27.68 \%, 25.56 \%, 28 \%$ and $37.2 \%$ at 3, 6, 9 and 12 months. Ulceration was associated with active osteomyelitis in $7.0 \%$ of the total population (288) at registration, and in $10.2 \%, 9.9 \%, 7 \%$ and $10.1 \%$ at 3, 6, 9 and 12 months.

Data on the provision of off-loading (other than orthotic shoes) at the initial visit were available for 246 patients (85.4\%). Initial off-loading was with a non-removable device in only $35.4 \%$ (below knee $33.7 \%$, foot only $1.7 \%$ ) of patients and with a removable device in $50 \%$ of cases (below knee $47.2 \%$, foot 2.8\%). However, $40.1 \%$ 
used non-removable and $65.2 \%$ removable off-loading devices at some stage during the follow-up period. Treatment with bisphosphonates was given intravenously in $25.4 \%$ patients and orally in $19.4 \%$. The median time from presentation to clinic and treatment with intravenous bisphosphonates was 2 weeks (range 0 to 79). Five patients had osteotomies, 13 had either internal or external fixation, and there were 13 minor and nine major (above ankle) amputations.

In the 219 patients for whom a resolution date was available, there was a significant association between the type of off-loading used and the median (range) time to resolution, this being 9 (3-25) months in those whose initial off-loading was with a non-removable off-loading device, versus 12 (3-36) months in those who had never had a non-removable cast (Mann-Whitney $U=3229, p=$ 0.001). The median (range) time to resolution in patients who had received bisphosphonates either intravenously or orally was 12 (3-39)months, which was significantly longer than in patients receiving neither (10 [2-29]months; Mann-Whitney $U=4496, p=0.005$ ). There appeared to be no interaction between the type of off-loading used and the use of bisphosphonates ( $p=0.194$ ANOVA).

\section{Discussion}

As far as we are aware, this is the largest observational survey of patients with acute Charcot foot in diabetes. The median time to resolution in this survey was longer than that reported in single-centre cohorts [11-14], one possible explanation being that we had predefined resolution as the patient being ambulant in normal or orthotic footwear. As the measuring, ordering, manufacture, fitting and 'breaking-in' of such footwear can take several weeks, it is possible that the actual time to resolution was rather shorter than reported here. An agreed standard is required for future studies.

It is accepted that the treatment of choice for acute Charcot foot involves the provision of off-loading in a nonremovable device. Nevertheless, it is surprising that only $35 \%$ of the patients were off-loaded with non-removable devices at baseline and only $40 \%$ had a non-removable device at any stage in the follow-up period. Overall, however, the findings of this survey suggest that the use of non-removable off-loading could shorten the median time to resolution by approximately 3 months.

The effectiveness of bisphosphonates in the management of acute Charcot foot remains to be substantiated. Only $25 \%$ of the patients in this series were reported to have been given intravenous bisphosphonate treatment, with almost 20\% receiving oral treatment (although not necessarily always for Charcot's disease) and some having both. It was, however, concerning to find that the use of bisphosphonates was associated with a longer time to resolution in this study. A very recent pilot RCT suggests that the use of zoledronic acid infusion is indeed associated with a significant prolongation of the time to resolution [15].

Any conclusions from this observational study must be drawn with care. The study was uncontrolled and potentially influenced by selection bias, even though participating centres were asked to include every case of new and active Charcot foot cared for after the first case was registered. It is also possible that reliance on clinician judgement for the diagnosis could have resulted in heterogeneity of the selected population. Our findings are also limited by the fact that data on the time to resolution were available in only $76 \%$ of the total population. One possible explanation of the prolonged time to resolution following bisphosphonate treatment could be that this treatment may have been reserved for patients judged to be more severe or for those not responding to off-loading. However, this seems unlikely, because the median time from first clinic appointment to the administration of bisphosphonates was only 2 weeks. It is also possible that some clinicians may have opted for bisphosphonates if they were unable to provide non-removable off-loading, but we observed no statistical interaction with type of off-loading (data not shown). The effects of off-loading and of bisphosphonate use can only be answered using appropriately powered randomised placebo-controlled trials with robust, long-term and clinically relevant outcome measures.

Acknowledgements We thank V. Savage (Foot Ulcer Trials Unit, Nottingham University Hospitals NHS Trust) for all her help in the preparation and conduct of this study. We also thank Diabetes UK for funding the study (project grant 04/0002935), as well as S. Breed (SB Communications Group) and the Diabetic Foot Journal for helping to promote it. We also thank G. Watson (Department of Computer Sciences, University of Nottingham) for writing the software, and all the clinicians who registered their patients.

Contribution statement FLG, GRJ, MEE, EJB, GR and WJJ planned the study. FLG, RC and WJJ supervised the conduct of the study, data capture and analysis. FLG and WJJ prepared and revised the manuscript, and all authors contributed to and approved the final manuscript.

Duality of interest The authors declare that there is no duality of interest associated with this manuscript.

\section{References}

1. Rajbhandari SM, Jenkins RC, Davies C, Tesfaye S (2002) Charcot neuroarthropathy in diabetes mellitus. Diabetologia 45:1085-1096

2. Jeffcoate WJ, Lima J, Nobrega L (2000) The Charcot foot. Diabet Med 17:253-258

3. Frykberg RG, Kozak GP (1978) Neuropathic arthropathy in the diabetic foot. Am Fam Physician 17:105-113 
4. Jeffcoate WJ, Game F, Cavanagh PR (2005) The role of proinflammatory cytokines in the cause of neuropathic osteoarthropathy (acute Charcot foot) in diabetes. Lancet 366:2058-2061

5. Jeffcoate WJ, Game FL (2010) New theories on the cause of the Charcot foot of diabetes. In: Frykberg R (ed) The Charcot foot and ankle DTP. Towson, Maryland, pp 29-43

6. Uccioli L, Sinistro A, Almerighi C et al (2010) Proinflammatory modulation of the surface and cytokine phenotype of monocytes in patients with acute Charcot foot. Diabetes Care 33:350-355

7. Pitocco D, Zetano G, Gioffre G et al (2009) Association between osteoprotegerin G1181 and T245G polymorphisms and diabetic Charcot neuroarthropathy. Diabetes Care 32:1694-1697

8. Sinacore DR (1996) Acute Charcot arthropathy in patients with diabetes mellitus: healing times by foot location. J Diabetes Complications 12:287-293

9. Pinzur MS, Lio T, Posner M (2006) Treatment of Eichenholtz stage I Charcot foot arthropathy with a weightbearing total contact cast. Foot Ankle Int 27:324-329
10. Pakarinen TK, Laine HJ, Honkonen SE, Peltonen J, Oksala H, Lahtela J (2002) Charcot arthropathy of the diabetic foot. Current concepts and review of 36 cases. Scand J Surg 91:195-201

11. Armstrong DG, Todd WF, Lavery LA, Harkless LB, Bushman TR (1997) The natural history of acute Charcot's arthropathy in a diabetic foot specialty clinic. Diabet Med 14:357-363

12. Selby PL, Young MJ, Boulton AJM (1993) Bisphosphonates: a new treatment for diabetic Charcot neuroarthropathy. Diabet Med $11: 28-31$

13. Jude EB, Selby PL, Burgess J et al (2001) Bisphosphonates in the treatment of Charcot neuroarthropathy: a double blind randomised controlled trial. Diabetologia 44:2032-2037

14. Pitocco D, Rutolo R, Caputo S et al (2005) Six month treatment with alendronate in acute Charcot neuropathy. Diabetes Care 28:1214-1215

15. Pakarinen TK, Laine HJ, Mäenpää H, Mattrila P, Lahtela J (2011) The effect of zoledronic acid on the clinical resolution of Charcot neuroarthropathy: a pilot randomized controlled trial. Diabetes Care 34:1514-1516 